\title{
Modeling Returns and Unconditional Variance in Risk Neutral World for Liquid and Illiquid Market
}

\author{
Ivivi Joseph Mwaniki \\ School of Mathematics, Statistics, Actuarial and Finance Division, University of Nairobi, Nairobi, Kenya \\ Email: jimwaniki@uonbi.ac.ke
}

Received 6 January 2015; accepted 25 January 2015; published 28 January 2015

Copyright @ 2015 by author and Scientific Research Publishing Inc.

This work is licensed under the Creative Commons Attribution International License (CC BY). http://creativecommons.org/licenses/by/4.0/

(c) (i) Open Access

\begin{abstract}
This article seeks to model daily asset returns using log-ARCH-Lévy type model which is expected to reproduce most of the stylized features of financial time series data (such as volatility clustering, leptokurtic nature of log returns, joint covariance structure and aggregational Gaussianity) that are empirically found in different types of market. In addition, unconditional variance of daily log returns in risk neutral world of different conditional heteroscedastic models is derived. A key observation is that liquid markets and illiquid market may not have the same underlying dynamics. For instance empirical analysis based on S\&P500 index log returns as a liquid market do not have autoregressive part in their first moments while in Nairobi Securities Exchange NSE20 index there is strong presence of autoregressive dynamics of order three, i.e. AR(3). Higher moments of both markets are serially correlated.
\end{abstract}

\section{Keywords}

AR-APARCH, Lévy Increments, Generalized Hyperbolic Distribution, Normal Inverse Gaussian, Illiquid Market

\section{Introduction}

It is well known that the stock price changes are neither independent nor identically distributed. There are linear and nonlinear dependencies between successive price changes. Distributional assumptions concerning risky asset log returns play a key role in option pricing. According to research finding of Mandelbrot [1], evidence indicates that the empirical distributions of daily stock returns differ significantly from the traditional Gaussian model. In search of satisfactory descriptive models for financial data, large number of distributions have been 
tried (see for example, [2]-[6]).

The deviations from normality become more severe when more frequent data are used to calculate stock returns. Various studies have shown that the normal distribution does not accurately describe observed stock return data. Over the past several decades, some stylized facts have emerged about the statistical behavior of speculative market returns such as aggregational Gaussianity, volatility clustering, etc see [7] [8]. On the same note, most of the literature for example [9]-[12] and references therein, assume that daily log returns, can be modeled by exponential Lévy processes and geometric Lévy process.

There are two important directions in the literature regarding these type of stochastic volatility models. Continuous-time stochastic volatility process represented in general by a bivariate diffusion process, and the discrete time autoregressive conditionally heteroscedastic (ARCH) model of [13] or its generalization (GARCH) as first defined by [14]. Option pricing in GARCH models has been typically done using the local risk neutral valuation relationship (LRNVR) pioneered by [15]. The crucial assumptions in his construction are the conditional, normal distribution of the asset returns under the underlying probability space and the invariance of the conditional volatility to the change of measure. The empirical performance of these normal option pricing models has been studied extensively, for example in [16], [17].

The main focus of this paper is to develop a ARCH type Lévy model which attempts to capture some of the stylized features observed in demeaned log returns from any market data. More so we derive unconditional variance of daily log returns in risk neutral world of different ARCH type models, and an in-depth empirical study in liquid and illiquid market. All parameters are estimated from historical data, i.e. for S\&P500 index from January 3, 1990 to January 18, 2008 and NSE20 index from March 2, 1998 to July 11, 2007.

The article is organized as follows. Section 2 provides a brief overview of ARCH type models and Lévy increments resulting to parameter estimation of observed salient features. In Section 3 which is our major contribution, unconditional variance of different ARCH type models is presented. Filtered Leptokurtic residuals of Lévy increments are calibrated. Conclusions are drawn in Section 4. Appendix is in the last section.

\section{ARCH Type Models}

ARCH-type models are in general, discrete models used to estimate volatility of financial time series data such stock returns, interest rates and foreign exchange rates. Let

$$
r_{t}=\log \frac{S_{t}}{S_{t-1}}-\mathbb{E} \log \frac{S_{t}}{S_{t-1}}
$$

where $S_{t}$ denotes the price of stock at time $t$. Define the following equation

$$
r_{t}=\mu_{t}+\varepsilon_{t} \quad \varepsilon_{t} \sim N\left(0, \sigma_{t}^{2}\right)
$$

where

$$
\sigma_{t}^{2}=\omega+\sum_{i=1}^{p} \alpha_{i} \varepsilon_{t-i}^{2}+\sum_{j=1}^{q} \beta_{j} \sigma_{t-j}^{2}, t=1, \cdots, T .
$$

where $\sigma_{t}^{2}$ is the $\operatorname{GARCH}(p, q)$ volatility process. If $q=0$ then $\sigma_{t}$ is $\operatorname{ARCH}(p)$. [18] and [19] provide a general specifications of volatility dynamic that nest most ARCH type models. In this connection volatility dynamics can be written as

$$
\sigma_{t}^{2}=\omega+\beta \sigma_{t-1}^{2}+\alpha \sigma_{t-1}^{2} f\left(z_{t-1}\right)
$$

where $f\left(z_{t-1}\right)$ is the innovation function. Different GARCH models are mainly characterized by the following specifications of the innovation function $f\left(z_{t-1}\right)$.

$$
f\left(z_{t-1}\right)= \begin{cases}z_{t-1}^{2}, & \text { Simple; } \\ \left(z_{t-1}-\theta\right)^{2}, & \text { Leverage; } \\ \left\{\left|z_{t-1}-\theta\right|-k\left(z_{t-1}-\theta\right)^{2}\right\}, & \text { News; } \\ \left(z_{t-1}-\theta\right)^{2 \gamma}, & \text { Power; } \\ \left\{\left|z_{t-1}-\theta\right|-\kappa\left(z_{t-1}-\theta\right)^{2 \gamma}\right\}, & \text { News and power; }\end{cases}
$$


The innovation function is used to model asymmetry and news impact to say the least. These GARCH models can be generalized to allow non-linearity of volatility dynamics by using Box-Cox transformation as follows

$$
\sigma_{t}^{\psi}=\omega+\beta \sigma_{t-1}^{\psi}+\alpha \sigma_{t-1}^{\psi} f\left(z_{t-1}\right) \text {, with } f\left(z_{t-1}\right)=\left(z_{t-1}-\theta\right)^{2 \psi}
$$

which implies modeling news and power, will nest most of the proposed GARCH models in Literature. Note that the leverage parameter $\theta$ shifts the innovation function, the news parameter $\kappa$ tilts the innovation, and the power parameters $\gamma$ and $\psi$ flatten or steepen the innovation function. Such a model (4) is the Asymmetric Power Autoregressive Conditional Heteroscedastic model i.e. APARCH model defined in (5).

The $\operatorname{APARCH}(m, n)$ model of can be written as follows

$$
\begin{gathered}
X_{t}=\varepsilon_{t}, \quad \varepsilon_{t}=\sigma_{t} z_{t}, z_{t} \sim \text { i.i.d }(0,1) \\
\sigma_{t}^{\delta}=\omega+\sum_{i=1}^{m} \alpha_{i}\left(\left|\varepsilon_{t-i}\right|-\gamma_{i} \varepsilon_{t-i}\right)^{\delta}+\sum_{j=1}^{n} \beta_{j} \sigma_{t-j}^{\delta}
\end{gathered}
$$

subject to $\omega>0, \delta \geq 0, \alpha_{i} \geq 0,-1<\gamma<1$, for $i=1, \cdots, m, \quad \beta_{j} \geq 0$, for $j=1, \cdots, n$. and

$$
\sum_{i}^{m} k_{i}+\sum_{j}^{n} \beta_{j}<1 \text {, where } k_{i}=\alpha_{i}\left(\left|\varepsilon_{t-i}\right|-\gamma_{i} \varepsilon_{t-i}\right)^{\delta}
$$

The model introduces a Box-Cox power transformation on the conditional standard deviation process and on the asymmetric innovations, $\alpha_{i}\left(\left|\varepsilon_{t-i}\right|-\gamma_{i} \varepsilon_{t-i}\right)^{\delta}$, adds flexibility of a varying exponent with an asymmetry coefficient to take the leverage effect into account. The properties of APARCH model have been studied, see [20]. The model nests seven other ARCH extensions as special cases.

- ARCH model of [13] when $\delta=2, \gamma_{i}$, and $\beta_{j}=0$;

- GARCH model of [14] when $\delta=2$, and $\gamma_{i}=0$;

- GJR-GARCH Model of [21] when $\delta=2$;

- TARCH Model of [22] when $\delta=1$.

Note that $\mu_{t}=\mathbb{E}\left(r_{t} \mid \mathfrak{F}_{t-1}\right)$ denote the conditional mean given the information set $\mathfrak{F}_{t-1}$ available at time $t-1$. The innovation process for the conditional mean is then given by $\varepsilon_{t}=r_{t}-\mu_{t}$ with corresponding unconditional variance $\sigma^{2}$ and zero unconditional mean. The conditional variance is defined as $\sigma_{t}=V\left(r_{t} \mid \mathfrak{F}_{t-1}\right)$.

\subsection{Empirical Data}

For simplicity, we focus on daily closing indices $\left\{S_{t}\right\}$ as reported in Nairobi Securities Exchange for NSE20 share index and S\&P500 index in New-York Stock Exchange. Daily log-returns $X_{t}$ of S\&P500 index are computed from January 3, 1990 to January 18, 2008 for a total of 4550 daily observations. While for NSE20, share indexes are computed from March 2, 1998 to July 11, 2007 for a total of 2317 daily observations.

All return series exhibit strong conditional heteroscedasticity. The Ljung and Box test rejects the hypothesis of homoscedasticity at all common levels both for returns in S\&P500 index and AR(3) residuals of linear regression in NSE20 share index. We estimate GARCH type models assuming conditional normality. With respect to the absolute value of parameter estimates, we find that $(0<\alpha+\beta<1)$ but different for both indices (NSE20 $(0<\alpha+\beta=0.924238<1)$, S\&P500 $(0<\alpha+\beta=0.994097<1)$ ), indicating the typical higher persistence of shocks in volatility in New York Stock exchange compared to Nairobi Securities Exchange. Model (5) is estimated using Pseudo Maximum Likelihood estimator based on the assumption of conditional normal innovations. The parameter estimates of (8) are reported in Table 1 and AR-ARCH residual calibrations of GH distribution (9) are presented in Table 2. Empirical and kernel densities of fitted distributions for both indices are compared in Figure 1.

$$
\begin{gathered}
X_{t}=\phi_{1} X_{t-1}+\phi_{2} X_{t-2}+\phi_{3} X_{t-3}+\varepsilon_{t}, \varepsilon_{t}=\sigma_{t} Z_{t}, Z_{t} \sim N(0,1), \\
\sigma_{t}^{\delta}=\omega+\sum_{i=1}^{m} \alpha_{i}\left(\left|\varepsilon_{t-i}\right|-\gamma_{i} \varepsilon_{t-i}\right)^{\delta}+\sum_{j=1}^{n} \beta_{j} \sigma_{t-j}^{\delta}
\end{gathered}
$$

\subsection{Lévy Increments}

Suppose $\phi(u)$ is the characteristic function of a distribution. If for every positive integer $n, \phi_{n}(u)$ is the 
Table 1. GARCH and GJR model estimates for the indices.

\begin{tabular}{|c|c|c|c|c|}
\hline & NSE20 & & S\&P500 & \\
\hline Parameter & GARCH & $\operatorname{GJR}(\delta=2)$ & GARCH & $\operatorname{GJR}(\delta=2)$ \\
\hline$\phi_{1}$ & $0.18915(0.024496)$ & $0.18136(0.02424)$ & & \\
\hline$\phi_{2}$ & $0.16451(0.023785)$ & $0.16245(0.02352)$ & & \\
\hline$\phi_{3}$ & $0.11388(0.023413)$ & $0.11516(0.02308)$ & & \\
\hline$\omega \times 10^{4}$ & $0.03549(0.006902)$ & $0.03458(0.00647)$ & $0.006577(0.001645)$ & $0.01088(0.00204)$ \\
\hline$\alpha$ & $0.15023(0.017978)$ & $0.18578(0.02528)$ & $0.056461(0.0067528)$ & $0.00322(0.00512)$ \\
\hline$\beta$ & $0.78763(0.024753)$ & $0.79045(0.02373)$ & $0.937566(0.0074845)$ & $0.93202(0.0079)$ \\
\hline $\operatorname{GJR}(\gamma)$ & & $-0.07332(0.02592)$ & & $0.10558(0.0123)$ \\
\hline$Q(10)$ & $9.3468(0.2287)$ & $8.8337(0.2648)$ & $16.5309(0.08541)$ & $15.2862(0.1220)$ \\
\hline$Q^{2}(10)$ & 7.1689 (0.5739) & $8.46159(0.38973)$ & $6.8918(0.54835)$ & $5.9298(0.6551)$ \\
\hline $\operatorname{lgl}$ & -8363.5 & -8367.7 & -15090.9 & -15090.9 \\
\hline$n$ & 2316 & 2316 & 4549 & 4549 \\
\hline
\end{tabular}

Notes: standard errors are in parenthesis. lgl is the log likelihood.

$n^{\text {th }}$ power of a characteristic function, we say that the distribution is infinitely divisible. One can define for every such infinitely divisible distribution a stochastic process $X=\left\{X_{t}, t \geq 0\right\}$ called a Lévy process, which starts at zero, has independent and stationary increments and such that the distribution of an increment over $[s, s+t], s, t \geq 0$ has $(\phi(u))^{t}$ is the characteristic function. For more detailed treatment of Lévy process, see [23].

Definition 2.1 The probability density function of the one-dimensional Generalized Hyperbolic distribution is given by the following:

$$
f_{G H}(x ; \alpha, \beta, \delta, \mu, \lambda)=\frac{(\gamma / \delta)^{\lambda}}{\sqrt{2 \pi} K_{\lambda}(\delta \gamma)} \frac{K_{\lambda-\frac{1}{2}}\left(\alpha \sqrt{\delta^{2}+(x-\mu)^{2}}\right)}{\left(\sqrt{\delta^{2}+(x-\mu)^{2}} / \alpha\right)^{\frac{1}{2}-\lambda}} \mathrm{e}^{\beta(x-\mu)}
$$

where $\gamma^{2}=\alpha^{2}-\beta^{2}$ and $K_{\lambda}$ is the modified Bessel function of third kind, with the index $\lambda$.

$$
K_{\lambda}(\omega)=\frac{1}{2} \int_{0}^{\infty} \exp \left[-\frac{\omega}{2}\left(v^{-1}+v\right)\right] v^{\lambda-1} \mathrm{~d} v
$$

$\mu$ is the location parameter and can take any real value, $\delta$ is a scale parameter; $\alpha$ and $\beta$ determine the distribution shape and $\lambda$ defines the subclasses of GH and is related to the tail flatness.

The mean and variance of GH distribution are given respectively by the followings

$$
E(X)=\mu+\frac{\beta \delta}{\sqrt{\alpha^{2}-\beta^{2}}} \frac{K_{\lambda+1}(\zeta)}{K_{\lambda}(\zeta)}
$$

and

$$
\operatorname{Var}(X)=\delta^{2}\left(\frac{K_{\lambda+1}(\zeta)}{\zeta K_{\lambda}(\zeta)}+\frac{\beta^{2}}{\alpha^{2}-\beta^{2}}\left[\frac{K_{\lambda+2}(\zeta)}{K_{\lambda}(\zeta)}-\left(\frac{K_{\lambda+1}(\zeta)}{K_{\lambda}(\zeta)}\right)^{2}\right]\right)
$$

where $\zeta=\delta \sqrt{\alpha^{2}-\beta^{2}}$. Note that, if $X \sim G H(\lambda, \alpha, \beta, \delta, v)$, then

$$
X \sim G H\left(-\frac{1}{2}, \alpha, \beta, \delta, \mu\right) \text { has normal-Inverse Gaussian distribution (NIG) }
$$




$$
\begin{aligned}
& X \sim G H(1, \alpha, \beta, \delta, \mu) \text { hyperbolic distribution (HY) } \\
& X \sim G H(\lambda, \alpha, \beta, 0, \mu) \text { variance-gamma distribution (VG) } \\
& f_{G H}(x ; \alpha, \beta, \delta, \mu, \lambda)=\frac{(\gamma / \delta)^{\lambda}}{\sqrt{2 \pi} K_{\lambda}(\delta \gamma)} \frac{K_{\lambda-\frac{1}{2}}\left(\alpha \sqrt{\delta^{2}+(x-\mu)^{2}}\right)}{\left(\sqrt{\delta^{2}+(x-\mu)^{2}} / \alpha\right)^{\frac{1}{2}-\lambda}} \mathrm{e}^{\beta(x-\mu)} \\
& f_{N I G}(x ; \alpha, \beta, \delta, \mu)=\frac{\alpha}{\pi} \exp \left(\delta \sqrt{\alpha^{2}-\beta^{2}}+\beta(x-\mu)\right) \frac{K_{1}\left(\alpha \delta \sqrt{1+z^{2}}\right)}{\sqrt{1+z^{2}}}
\end{aligned}
$$

For more information about GH distribution, see [24].

\section{Modeling the Underlying}

Let $\left(\Omega, \mathcal{F},\left(\mathfrak{F}_{t}\right)_{t \in[0, T]}, \mathbb{P}\right)$ be a stochastic basis describing the uncertainty of the economy. We refer to $\mathbb{P}$ as the physical probability measure and $\mathfrak{F}_{t}$ represent the information flow driven by Brownian motion $B=\left(B_{t}\right)_{t \in[0, T]}$ and Lévy proces $L=\left(\mathfrak{L}_{t}\right)_{t \in[0, T]}$. Let $S_{t}$ be the price of a stock at time $t$ adapted to the natural filtration $\mathfrak{F}_{t}$. Define daily $\log$ return as $X_{t}=\log S_{t}-\log S_{t-1}$. It is well known from our empirical studies that $X_{t}$ can he represented as $X_{t}=\mu_{t}+\varepsilon_{t}+\xi_{t}$ where $\mu_{t}$ is a mean function and $\varepsilon_{t}, \xi_{t}$ are the two components of the error term. Moreover, define a $p^{\text {th }}$ order autoregressive process $\left\{X_{t}\right\}$ with $\operatorname{APARCH}(m, n)$ error as

$$
\begin{aligned}
& X_{t}=\mu_{t}+\varepsilon_{t}+\xi_{t} \\
& \mu_{t}=\sum_{r=1}^{p} \phi_{r} X_{t-r}+\mu, t \in \mathcal{Z}^{+} \\
& \varepsilon_{t}+\xi_{t}=\sigma_{t}\left(Z_{t}+\sigma \mathfrak{L}_{t}\right), Z_{t}, \text { and } \mathfrak{L}_{t} \sim \text { i.i.d }(0,1), Z_{0}=0, \mathfrak{L}_{0}=0 \\
& \sigma_{t}=\operatorname{APARCH}(m, n), m, n \in \mathbb{Z}^{+}
\end{aligned}
$$

where $Z_{t}$ and $\mathfrak{L}_{t}$ are identically and independently distributed random variables. A general time series model for $\log$ returns would be

$$
X_{t}=\mu_{t}+\sigma_{t}\left(Z_{t}+\sigma \mathfrak{L}_{t}\right), \quad Z_{t} \sim N(0,1), \mathfrak{L}_{t} \in G H
$$

\subsection{Risk Neutralization}

In this section, we construct risk neutral probability measure in the context of [15] and [19]. Duan [15] introduced the GARCH option pricing model by generalizing the traditional risk neutral valuation methodology to the case of conditional heteroscedasticity, the so called Local Risk Neutral Valuation Relationship (LRNVR).

Definition 3.1 A pricing measure $\mathbb{Q}$ is said to satisfy the locally risk-neutral valuation relationship (LRNVR) if measure $\mathbb{Q}$ is equivalent to $\mathbb{P}$, and

Table 2. Calibration of AR-GARCH(1,1) residuals to a class of infinitely divisible distributions.

\begin{tabular}{cccccccc}
\hline NSE20 & GH & HY & NIG & S\&P500 & GH & HY & NIG \\
\hline$\lambda$ & -1.79233 & 1.0000 & -0.5000 & $\lambda$ & 2.38336 & 1.0000 & -0.500 \\
$\alpha$ & 0.98225 & 1.15813 & 0.66862 & $\alpha$ & 0.14671 & 1.68640 & 1.33977 \\
$\beta$ & -0.05226 & -0.06604 & -0.05864 & $\beta$ & -0.14279 & -0.14976 & -0.15755 \\
$\delta$ & 1.79373 & 0.45207 & 1.18530 & $\delta$ & 0.04052 & 1.04004 & 1.59588 \\
$\mu$ & 0.12296 & 0.13923 & 0.13014 & $\mu$ & 0.14292 & 0.15130 & 0.16032 \\
\hline
\end{tabular}


Kernel Density of i.i.d. noise vs Fitted NM,GH

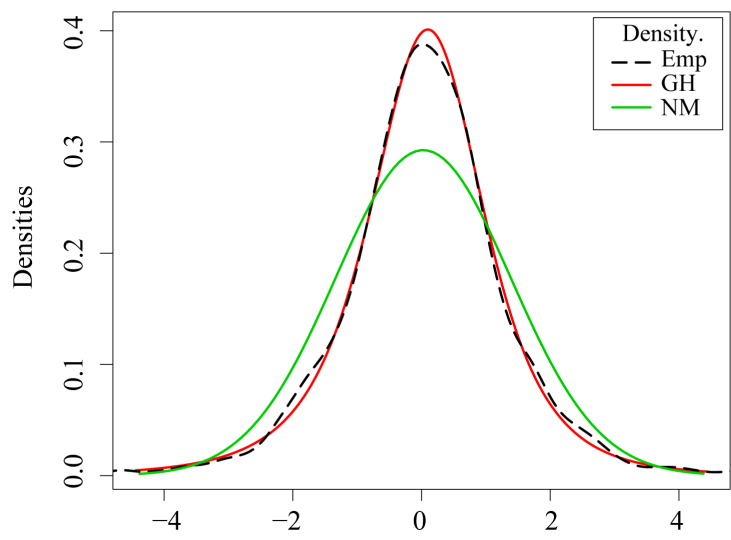

Kernel Density of i.i.d. noise vs Fitted NM,GH

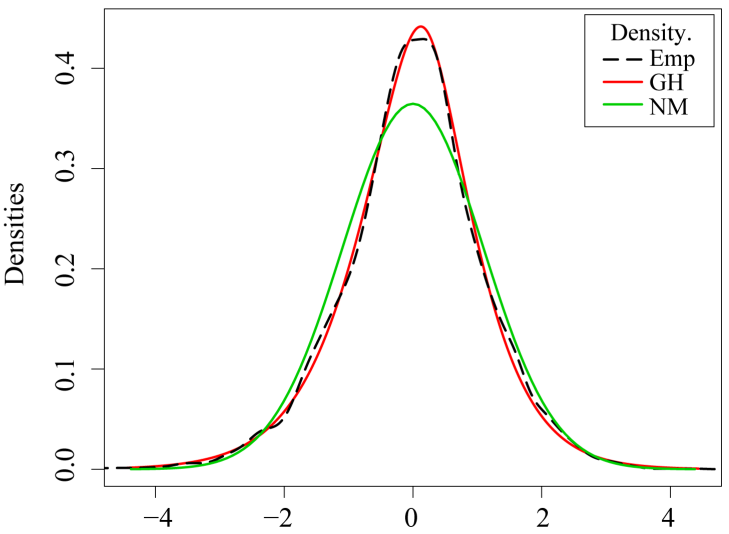

Figure 1. Empirical and kernel densities of standardized GARCH filtered Lévy increments of NSE20 index (left) S\&P500 index (right) calibrated vs. density of fitted infinitely divisible distributions and normal distributions.

$$
\begin{aligned}
& E^{\mathbb{Q}}\left[X_{t} \mid \mathfrak{F}_{t-1}\right]=r \\
& \operatorname{Var}^{\mathbb{Q}}\left(X_{t} \mid \mathfrak{F}_{t-1}\right)=\operatorname{Var}^{\mathbb{Q}}\left(X_{t} \mid \mathfrak{F}_{t-1}\right)
\end{aligned}
$$

almost surely with respect to measure $\mathbb{P}$.

For some commonly used assumptions concerning utility functions and distributions of change of consumption, [15] shows that a representative agent maximizes his expected utility using the LRNVR measure $\mathbb{Q}$. Risk neutralization should leave the variance unchanged and should transform the conditional expectation so that the discounted expected price of the underlying asset becomes a martingale. It is worth noting that in the case of homoscedasticity process, $(p=0, q=0)$, the conditional variances become the same constant and the LRNVR reduces to conventional risk neutral valuation relationship.

Consider the general model of daily log returns under the data generating probability measure $\mathbb{P}$ as

$$
X_{t}=\ln \left(S_{t} / S_{t-1}\right) \text {, where, }\left\{\begin{array}{l}
X_{t}=\mu_{t}+\varepsilon_{t}+\xi_{t} ; \\
\varepsilon_{t} \mid \mathfrak{F}_{t-1} \sim N\left(0, \sigma_{t}\right) ; \\
\sigma_{t}^{2}=\omega+\alpha \varepsilon_{t}+\beta \sigma_{t-1}^{2}
\end{array}\right.
$$

where the parameters $\omega>0, \alpha>0$ and $\beta>0$ and $1-\beta-\alpha>0$ and given $\sigma_{0}$. The sequence $\left\{\varepsilon_{t}\right\}$ and $\left\{\xi_{t}\right\}$ are conditionally independent, while $\mathfrak{F}_{t-1}$ is the past information set. $\mu_{t}$ represents the conditional expectation of returns.

The pricing measure $\mathbb{Q}$ shifts the error term $\varepsilon_{t}$ by some measurable function $\lambda_{t}$, so that the conditional expectation of $X_{t}$ becomes equal to $r$. In the case of AR(1)APARCH(1,1)-Lévy filter, we follow the [25] argument. Therefore under the equivalent martingale measure $\mathbb{Q}$ the model (16) translates to

$$
\begin{aligned}
X_{t}=\mu_{t}+\varepsilon_{1 t}+\varepsilon_{2 t} ; \\
=\mu_{t}+\sigma_{t}\left(Z_{t}-\lambda_{1 t}\right)+\sigma_{t}\left(\mathfrak{L}_{t}-\lambda_{2 t}\right),\left\{\begin{array}{l}
\mu_{t}=v+\phi X_{t-1} ; \\
\lambda_{1 t}=\left(\mu_{t}-r\right) / \sigma_{t} ; \\
\lambda_{2 t}=\mathbb{E}_{t} ; \\
\sigma_{t}^{2}=f\left(\sigma_{s}^{2}, Z_{s}, \lambda_{1 s} ;-\infty<s<t\right) ;
\end{array}\right.
\end{aligned}
$$

The LRNVR implies that under the risk neutral measure $\mathbb{Q}$ the return process evolves as

$$
X_{t}=r+\sigma_{t}\left(Z_{t}+\mathfrak{L}_{t}-\mathbb{E} \mathfrak{L}_{t}\right),\left\{\begin{array}{l}
Z_{t} \sim N(0,1), \mathfrak{L}_{t} \sim N I G(\Theta) ; \\
\Theta=\left(\alpha_{N I G}, \beta_{N I G}, \mu_{N I G}, \delta_{N I G}\right) ;
\end{array}\right.
$$




$$
\begin{aligned}
& \sigma_{t}^{2}=\omega+\alpha\left(Z_{t-1}-\lambda_{t-1}\right)^{2} \sigma_{t-1}^{2}+\beta \sigma_{t-1}^{2}, \\
& \lambda_{t-1}=\left(\mu_{t-1}-r\right) / \sigma_{t-1}, \\
& \mu_{t-1}=v+\phi X_{t-2},
\end{aligned}
$$

It follows quite easily that

$$
\mathbb{E}^{\mathbb{Q}}\left[X_{t} \mid \mathfrak{F}_{t-1}\right]=r \text { and } \operatorname{Var}^{\mathbb{Q}}\left(X_{t} \mid \mathfrak{F}_{t-1}\right)=\operatorname{Var}^{\mathbb{Q}}\left(X_{t} \mid \mathfrak{F}_{t-1}\right)=\sigma_{t}^{2}\left(1+\operatorname{Var}^{\mathbb{Q}} \mathfrak{L}_{t}\right)
$$

\subsection{Unconditional Variance}

The following propositions provide the unconditional variance for the process $X_{t}$ under $\mathbb{Q}$

Proposition 3.1 Consider $A R(3) A P A R C H(1,1)$ Lévy filter, with $\delta=2$ and $k=0$ which implies $A R(3)$ $\operatorname{GARCH}(1,1)$ Lévy model, the unconditional variance of $X_{t}$ under the LRNVR equivalent measure $\mathbb{Q}$ is

$$
\operatorname{Var}^{\mathbb{Q}} X_{t}=\frac{\left(1+\operatorname{Var}_{t}\right)\left(\omega+\alpha\left[v-r\left(1-\sum_{j=1}^{3} \phi_{j}\right)\right]^{2}+2 r \sum_{i \neq j}^{3} \phi_{j} \phi_{i}\right)}{1-\alpha\left[1+\left(1+\operatorname{Var} \mathfrak{L}_{t}\right)\left(\sum_{j=1}^{3} \phi_{j}^{2}\right)\right]-\beta}
$$

Proof: See Appendix.

Proposition 3.2 A special case of AR(1)GARCH(1,1)Lévy filter the unconditional variance under the LRNVR equivalent measure $\mathbb{Q}$ is given by

$$
\operatorname{Var}^{\mathbb{Q}} X_{t}=\frac{\left(1+\operatorname{Var} \mathfrak{L}_{t}\right)\left[\omega+\alpha(v-r(1-\phi))^{2}\right]}{1-\alpha\left(1+\phi^{2}\left(1+\operatorname{Var} \mathfrak{L}_{t}\right)\right)-\beta}
$$

Proof: See Appendix.

Example 3.1 In case of Hyperbolic distribution we substitute mean and variance respectively into (25). Where the parameters used maximize the likelihood function of Hyperbolic distribution. i.e. Let

$\zeta_{H P}=\delta_{H P} \sqrt{\alpha_{H P}^{2}-\beta_{H P}^{2}}$ then,

$$
\begin{aligned}
\mathbb{E} \mathfrak{L}_{t} & =\mu_{H P}+\frac{\beta_{H P} \delta_{H P}}{\sqrt{\alpha_{H P}^{2}-\beta_{H P}^{2}}} \frac{K_{2}\left(\zeta_{H P}\right)}{K_{1}\left(\zeta_{H P}\right)} \text {, and } \\
& =0.0073397 \\
\operatorname{Var}_{t} & =\delta_{H P}^{2}\left(\frac{K_{2}\left(\zeta_{H P}\right)}{\zeta_{H P} K_{1}\left(\zeta_{H P}\right)}+\frac{\beta_{H P}^{2}}{\alpha_{H P}^{2}-\beta_{H P}^{2}}\left[\frac{K_{3}\left(\zeta_{H P}\right)}{K_{1}\left(\zeta_{H P}\right)}-\left(\frac{K_{2}\left(\zeta_{H P}\right)}{K_{1}\left(\zeta_{H P}\right)}\right)^{2}\right]\right) \\
& =1.713026
\end{aligned}
$$

Consider a discrete time economy, where interest rates and returns are paid after each time interval of equal spaced length. Suppose there is a price for risk, measured in terms of a risk premium that is added to the risk free interest rate $r$ to build the expected next period return. As in Duan [15], we adopt and extend the ARCH-M model of [26] with the risk premium being linear functional of the conditional standard deviation, hence the following model under $\mathbb{P}$,

$$
X_{t}=r+\lambda \sigma_{t}+\varepsilon_{t} \text { where } \begin{cases}\varepsilon_{t} \mid \mathfrak{F}_{t-1}=\sigma_{t}\left(Z_{t}+\mathfrak{L}_{t}\right), & \mathfrak{L}_{t} \text { infinitely divisible density; } \\ Z_{t} \sim N(0,1), & Z_{t} \text { Standard normal; } \\ \sigma_{t}^{2}=\omega+\left(\alpha \sigma_{t-1} Z_{t-1}\right)^{2}+\beta \sigma_{t-1}^{2}, & \operatorname{GARCH}(1,1) ;\end{cases}
$$

The parameters $\omega, \alpha$, and $\beta$ are constant parameters satisfying stationarity and positivity conditions, while 
the constant parameter $\lambda$ may be interpreted as the unit price for risk. If we change the function $\sigma_{t}^{2}$ in (29) to model news impact, we get threshold GARCH model of [21] where

$$
g(x)=\omega+\alpha_{1} x^{2} \mathbb{I}_{x<0}+\alpha_{2} x^{2} \mathbb{I}_{x \geq 0}
$$

hence the resulting TGARCH Lévy filter model

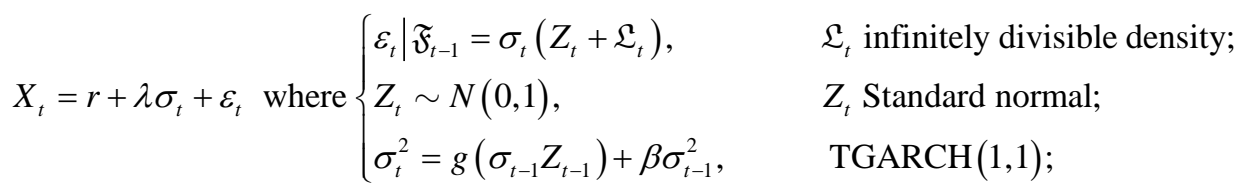

Proposition 3.3 The unconditional variance of the GARCH-M Lévy filter model under the LRNVR equivalent martingale measure $\mathbb{Q}$ is

$$
\operatorname{Var}^{\mathbb{Q}} X_{t}=\frac{\omega\left(1+\operatorname{Var}_{t}\right)}{1-\alpha\left(1+\lambda^{2}\right)-\beta}
$$

Proof: See Appendix.

Proposition 3.4 The unconditional variance of the TGARCH-M Lévy filter model under equivalent martingale measure $\mathbb{Q}$ is

$$
\operatorname{Var}^{\mathbb{Q}} X_{t}=\frac{\omega\left(1+\operatorname{Var}_{t}\right)}{1-\alpha_{1} \psi(\lambda)-\alpha_{2}\left(1+\lambda^{2}-\psi(\lambda)\right)-\beta}
$$

where

$$
\psi(u)=\frac{u}{\sqrt{2 \pi}} \exp \left(-\frac{1}{2} u^{2}\right)+\left(1+u^{2}\right) \Phi(u)
$$

and $\Phi(u)$ denoting the cumulative standard normal distribution function.

Proof: See Appendix.

\section{Concluding Remarks}

This article develops an log-ARCH-Lévy type risk neutral model. The proposed method delivers predictive distribution of the payoff function for a given econometric model. As a result, the probability distribution could be useful to market participants who wish to compare the model predictions to the potential prices in liquid and illiquid markets.

Any effective option pricing model is expected to be consistent with distributional and time series properties of the underlying asset. The proposed model accommodates most of the observed stylistic fact about financial time series data i.e. skewness and leptokurtic nature of demeaned GARCH filtered log returns and perhaps aggregational Gaussianity. In summary,

- developed markets and emerging markets may not have the same underlying dynamics. It would be incorrect to assume that a universal model for the underlying process for all markets.

- The presence of linear autoregressive dynamics AR(3)-GARCH(1,1) effects in NSE20 index affects the unconditional variance in risk neutral world. S\&P500 index was found to follow GARCH(1,1) plus leptokurtic residual which was calibrated in one class of generalized hyperbolic distributions,say for example, Normal inverse Gaussian (NIG).

- The presence of autoregressive dynamics, i.e. AR(3)-GARCH(1,1) model of NSE20 index as an example of illiquid market would have an impact in pricing options, if the index were to be used as an underlying process.

The log-ARCH-Lévy model is very tractable compared to other jump-diffusion or stochastic volatility models. It attempts to addresses the drawbacks of local volatilities. Further refinements and extensions are left for future research.

\section{Acknowledgements}

Comments from the Editor and the anonymous referee are acknowledged. Financial support from International Science Progam (Sweden)/EAUMP is greatly appreciated. 


\section{References}

[1] Mandelbrot, B. (1963) The Variation of Certain Speculative Prices. International Statistical Review, 36, 394-419.

[2] Clark, P. (1973) A Surbodinated Stochastic Process Model with Finite Variance for Speculative Prices. Econometrica, 41, 135-155. http://dx.doi.org/10.2307/1913889

[3] Madan, D. and Seneta, E. (1990) The Variance Gamma (V.G.) Model for Share Markets. Journal of Business, 63, 511524. http://dx.doi.org/10.1086/296519

[4] Eberlein, E. and Keller, U. (1995) Hyperbolic Distributions in Finance. Bernolli, 1, 281-299. http://dx.doi.org/10.2307/3318481

[5] Berndorff-Nielson, O. (1998) Process of Normal Inverse Gaussian Type. Finance and Stochastics, 2, 41-68. http://dx.doi.org/10.1007/s007800050032

[6] Mwaniki, I.J. (2010) On APARCH Lévy Filter Option Pricing Formula for Developed and Emerging Markets. PhD Thesis, University of Nairobi, Nairobi.

[7] Rydberg, T. (2000) Realistic Statistical Modeling of Financial Data. International Statistical Review, 68, $233-258$. http://dx.doi.org/10.1111/j.1751-5823.2000.tb00329.x

[8] Cont, R. (2001) Empirical Properties of Asset Returns: Stylized Facts and Statistical Issues. Quantitative Finance, 1, 223-236. http://dx.doi.org/10.1080/713665670

[9] Carr, P. and Madan, D. (1998) Option Valuation Using the Fast Fourier Transform. Journal of Computational Finance, 2, 61-73.

[10] Barndorff-Nielson, O. (1998) Process of Normal Inverse Gaussian Type. Finance Stochastics, 2, 41-68. http://dx.doi.org/10.1007/s007800050032

[11] Chan, T. (1999) Pricing Contingent Claims on Stock Driven by Lévy Processes. The Annals of Applied Probability, 9, 504-528. http://dx.doi.org/10.1214/aoap/1029962753

[12] Carr, P., German, H., Madan, D. and Yor, M. (2002) The Fine Structure of Asset Returns: An Empirical Investigation. Journal of Business, 75, 305-332. http://dx.doi.org/10.1086/338705

[13] Engle, R. (1982) Autoregressive Conditional Heteroscedasticity with Estimates of Variance of United Kingdom Inflation. Journal of Business and Economic Statistics, 9, 987-1008.

[14] Bollerslev, T. (1986) Generalized Autoregressive Conditional Heteroskedasticity. Journal of Econometrics, 31, 307327.

[15] Duan, J. (1995) The GARCH Option Pricing Model. Mathematical Finance, 5, 13-32. http://dx.doi.org/10.1111/j.1467-9965.1995.tb00099.x

[16] Härdle, W. and Hafner, C. (2000) Discrete Time Option Pricing with Flexible Volatility Estimation. Finance and Stochastics, 4, 189-207. http://dx.doi.org/10.1007/s007800050011

[17] Christoffersen, P. and Jacobs, K. (2004) Which GARCH Model for Option Valuation? Management Science, 50, 12041221. http://dx.doi.org/10.1287/mnsc.1040.0276

[18] Ding, Z., Granger, W. and Engle, R. (1993) A Long Memory Property of Stock Markets Returns and a New Model. Journal of Empirical Finance, 1, 83-106. http://dx.doi.org/10.1016/0927-5398(93)90006-D

[19] Hentschel, L. (1995) All in the Family Nesting Symmetric and Asymmetric GARCH Models. Journal of Financial Economics, 39, 71-104. http://dx.doi.org/10.1016/0304-405X(94)00821-H

[20] Laurent, S. (2004) Analytical Derivatives of the APARCH Model. Computational Economics, 24, 51-57. http://dx.doi.org/10.1023/B:CSEM.0000038851.72226.76

[21] Glosten, L., Jagannathan, R. and Runkle, D. (1993) The Relationship between Expected Value and the Volatility of the Nominal Excess Returns on Stocks. Journal of Finance, 48, 1779-1801. http://dx.doi.org/10.1111/j.1540-6261.1993.tb05128.x

[22] Zakoian, J. (1994) Threshold Heteroskedastic Models. Journal of Economic Dynamics and Control, 18, 931-955. http://dx.doi.org/10.1016/0165-1889(94)90039-6

[23] Sato, K. (1999) Lévy Process and Infinitely Divisible Distributions. Cambridge University Press, Cambridge.

[24] Barndorff-Nielsen, O. (1977) Exponentially Decreasing Distributions for Logarithms of Particle Size. Proceedings of the Royal Society London Series A, 353, 401-419.

[25] Hafner, C. and Herwartz, H. (2001) Option Pricing under Linear Autoregressive Dynamics, Heteroskedasticity, and Conditional Leptokurtosis. Journal of Empirical Finance, 8, 1-34. http://dx.doi.org/10.1016/S0927-5398(00)00024-4

[26] Engle, R., Lilian, D. and Robins, R. (1987) Estimating Time Varying Premia in Term Structure: The ARCH-M Model. Econometrica, 55, 391-407. http://dx.doi.org/10.2307/1913242 


\section{Appendix}

Proof of proposition 3.1

Given $X_{t}=r+\sigma_{t}\left(Z_{t}+\mathfrak{L}_{t}-\mathbb{E}_{\mathfrak{L}_{t}}\right) ; \lambda_{t}=\left(\mu_{t}-r\right) / \sigma_{t} ; \mu_{t}=v+\sum_{j=1}^{3} \phi_{j} y_{t-j}$ We note that $\mathbb{E}^{\mathbb{Q}} X_{t}=r$ and

$$
\begin{aligned}
& \mathbb{E}^{\mathbb{Q}}\left[X_{t}^{2}\right]=\mathbb{E}^{\mathbb{Q}}\left(r^{2}+2 r \sigma_{t} Z_{t}\left(\mathfrak{L}_{t}-\mathbb{E}_{\mathfrak{L}_{t}}\right)+\sigma_{t}^{2} Z_{t}^{2}\left(\mathfrak{L}-\mathbb{E}_{\mathfrak{L}}\right)^{2}\right)=r^{2}+\mathbb{E}^{\mathbb{Q}} \sigma_{t}^{2}\left(1+\operatorname{Var} \mathfrak{L}_{t}\right) \\
& \mathbb{E}^{\mathbb{Q}}\left[\sigma_{t}^{2}\right]=\omega+\alpha \mathbb{E}^{\mathbb{Q}}\left(Z_{t-1}-\lambda_{t-1}\right)^{2} \sigma_{t-1}^{2}+\beta \mathbb{E}^{\mathbb{Q}} \sigma_{t-1}^{2}=\omega+\alpha\left(\mathbb{E}^{\mathbb{Q}}\left[\sigma_{t-1}^{2}\right]+\mathbb{E}^{\mathbb{Q}}\left(\mu_{t}-r\right)^{2}\right)+\beta \mathbb{E}^{\mathbb{Q}} \sigma_{t-1}^{2}
\end{aligned}
$$

after rearranging and simple algebra

$$
\begin{aligned}
\mathbb{E}^{\mathbb{Q}}\left[\mu_{t-1}-r\right]^{2} & =v^{2}+\left(r^{2}+\mathbb{E}^{\mathbb{Q}} \sigma_{t-1}^{2}\left(1+\operatorname{Var} \mathfrak{L}_{t}\right)\right)\left(\sum_{j=1}^{3} \phi_{j}^{2}\right)-2 v r\left(1-\sum_{j=1}^{3} \phi_{j}\right)+r^{2}\left(1-\sum_{j=1}^{3} \phi_{j}\right)+\cdots \\
& =v^{2}+\left(r^{2}+\mathbb{E}^{\mathbb{Q}} \sigma_{t-1}^{2}\left(1+\operatorname{Var} \mathfrak{L}_{t}\right)\right)\left(\sum_{j=1}^{3} \phi_{j}\right)+r(1-2 v)\left(1-\sum_{j=1}^{3} \phi_{j}\right)+2 r^{2} \sum_{j \neq k}^{3} \phi_{j} \phi_{k}
\end{aligned}
$$

Thus under stationarity, the unconditional expectations are independent of $t$

$$
\mathbb{E}^{\mathbb{Q}}\left[\sigma_{t}^{2}\right]=\frac{\omega+r^{2}\left(\sum_{j=1}^{3} \phi_{j}\right)+r(1-2 v)\left(1-\sum_{j=1}^{3} \phi_{j}\right)+2 r^{2} \sum_{j \neq k}^{3} \phi_{j} \phi_{k}}{1-\alpha\left[1+\left(1+\operatorname{Var} \mathfrak{L}_{t}\right) \sum_{j=1}^{3} \phi_{j}^{2}\right]-\beta}
$$

Therefore, the unconditional variance of AR(3)GARCH(1,1)Levy filter model under LRNVR equivalent martingale measure is

$$
\begin{aligned}
\operatorname{Var}^{\mathbb{Q}} X_{t} & \frac{\left(1+\operatorname{Var}_{t}\right)\left(\omega+\alpha\left[v^{2}-2 v r\left(1-\sum_{j=1}^{3} \phi_{j}\right)+r^{2}\left(1-2 \sum_{j=1}^{3} \phi_{j}+\sum \phi_{j}^{2}\right)+2 r \alpha \sum_{j \neq k}^{3} \phi_{j} \phi_{k}\right]\right)}{1-\alpha\left[1+\left(1+\operatorname{Var} \mathfrak{L}_{t}\right)\left(\sum_{j=1}^{3} \phi_{j}\right)\right]-\beta} \\
= & \frac{\left(1+\operatorname{Var} \mathfrak{L}_{t}\right)\left(\omega+\alpha\left[v-r\left(1-\sum_{j=1}^{3} \phi\right)\right]^{2}+2 \alpha r \sum_{i \neq j}^{3} \phi_{j} \phi_{i}\right)}{1-\alpha\left[1+\left(1+\operatorname{Var}_{t}\right)\left(\sum_{j=1}^{3} \phi_{j}^{2}\right)\right]-\beta}
\end{aligned}
$$

Proof of proposition 3.2

This is a special case of (3.1) with $\phi_{1}=\phi$ and $\phi_{2}=\phi_{3}$.

Proof of proposition 3.3

It is a special case of proposition 3.4 when we take $\alpha_{1}=\alpha$ and $\alpha_{2}=0$.

Proof of proposition 3.4

Under measure $\mathbb{Q}$.

$$
X_{t}=r+\varepsilon_{t}=r+\sigma_{t}\left(\lambda+Z_{t}+\mathfrak{L}_{t}-\mathbb{E} \mathfrak{L}_{t}\right)
$$

where $\lambda$ is the risk premium and

$$
\begin{aligned}
& \sigma_{t}^{2}=\omega+\alpha_{1} \sigma_{t-1}^{2}\left(Z_{t-1}-\lambda\right)_{\mathbb{I}_{Z_{t-1}<0}}^{2}+\alpha_{2} \sigma_{t-1}^{2}\left(Z_{t-1}-\lambda\right)_{\mathbb{I}_{Z_{t-1}<0}}^{2} \\
& \operatorname{Var}^{\mathbb{Q}} X_{t}=\mathbb{E}^{\mathbb{Q}} y_{t}^{2}-r^{2}=r^{2}+\mathbb{E} \sigma_{t-1}^{2}\left(1+\operatorname{Var} \mathfrak{L}_{t}\right)-r^{2} \\
& \mathbb{E}^{\mathbb{Q}} \sigma_{t}^{2}=\omega+\alpha_{1} \psi(\lambda) \mathbb{E}^{\mathbb{Q}} \sigma_{t-1}^{2}+\alpha_{2}\left[1+\lambda^{2}-\psi(\lambda)\right] \mathbb{E}^{\mathbb{Q}} \sigma_{t-1}^{2}+\beta \mathbb{E}^{\mathbb{Q}} \sigma_{t-1}^{2} \\
& =\frac{\omega}{1-\alpha_{1} \psi(\lambda)-\alpha_{2}\left(1+\lambda^{2}-\psi(\lambda)\right)-\beta}
\end{aligned}
$$




$$
\text { thus } \operatorname{Var}^{\mathbb{Q}}\left(X_{t}\right)=\frac{\omega\left(1+\operatorname{Var}_{t}\right)}{1-\alpha_{1} \psi(\lambda)-\alpha_{2}\left(1+\lambda^{2}-\psi(\lambda)\right)-\beta}
$$

where, $\psi(u)=\frac{u}{\sqrt{2 \pi}} \exp \left(-\frac{1}{2} u^{2}\right)+\left(1+u^{2}\right) \Phi(u)$

and $\phi(u)$ denoting the cumulative standard normal distribution. Note that $Z_{t-1}^{\prime} \sim N(-\lambda, 1)$ and

$$
\begin{aligned}
& \mathbb{E}\left[Z_{t}^{\prime 2} \mathbb{I}_{Z_{t}^{\prime}<0} \mid \mathfrak{F}_{t-1}\right]=\frac{1}{\sqrt{2 \pi}} \int_{-\infty}^{0} Z^{2} \exp \left(-(Z+\lambda)^{2} / 2\right) \mathrm{d} z \\
& =\frac{1}{\sqrt{2 \pi}} \int_{-\infty}^{\lambda}(u-\lambda)^{2} \exp \left(-u^{2} / 2\right) \mathrm{d} u \\
& =\frac{1}{\sqrt{2 \pi}} \int_{-\infty}^{\lambda} u^{2} \exp \left(-u^{2} / 2\right) \mathrm{d} u-\frac{2 \lambda}{\sqrt{2 \pi}} \int_{-\infty}^{\lambda} u \exp \left(-u^{2} / 2\right) \mathrm{d} u+\frac{\lambda^{2}}{\sqrt{2 \pi}} \int_{-\infty}^{\lambda} \exp \left(-u^{2} / 2\right) \mathrm{d} u \\
& =\frac{-\lambda}{\sqrt{2 \pi}} \exp \left(-\lambda^{2} / 2\right)+\Phi(\lambda)+\frac{2 \lambda}{\sqrt{2 \pi}} \exp \left(-\lambda^{2} / 2\right)+\lambda^{2} \Phi(\lambda) \\
& =\frac{\lambda}{\sqrt{2 \pi}} \exp \left(-\frac{\lambda^{2}}{2}\right)+\left(1+\lambda^{2}\right) \phi(\lambda) \\
& =: \psi(\lambda)
\end{aligned}
$$

Therefore, for positive support

$$
\mathbb{E}^{\mathbb{Q}}\left[Z_{t}^{2} \mathbb{I}_{Z_{t} \geq 0} \mid \mathfrak{F}_{t-1}\right]=1+\lambda^{2}-\psi(\lambda)
$$


Scientific Research Publishing (SCIRP) is one of the largest Open Access journal publishers. It is currently publishing more than 200 open access, online, peer-reviewed journals covering a wide range of academic disciplines. SCIRP serves the worldwide academic communities and contributes to the progress and application of science with its publication.

Other selected journals from SCIRP are listed as below. Submit your manuscript to us via either submit@scirp.org or Online Submission Portal.
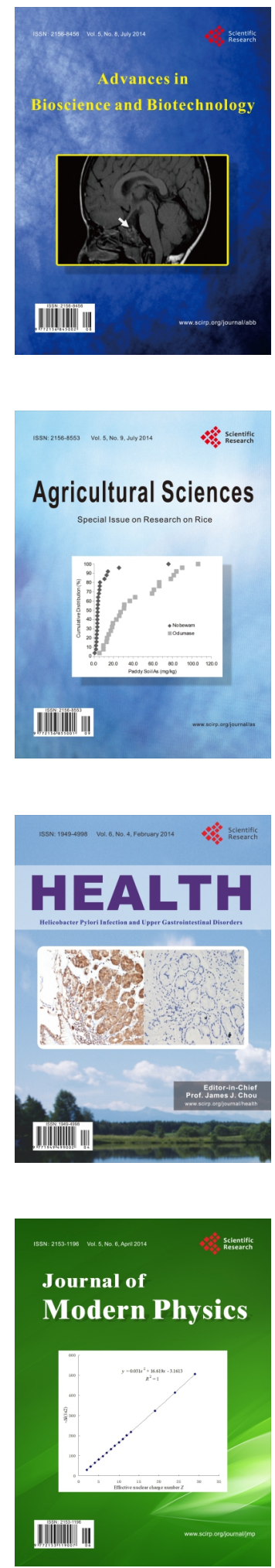
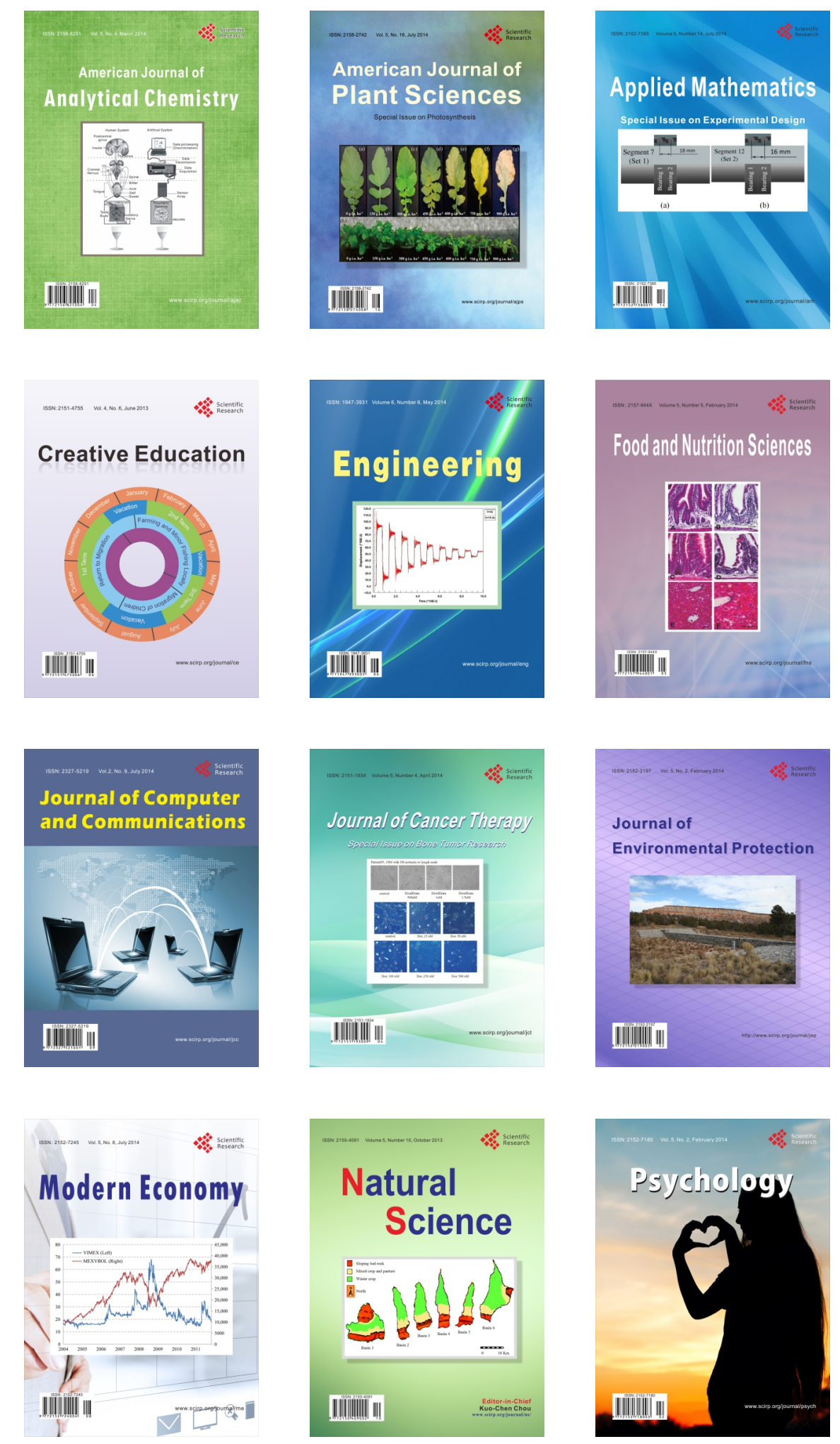